\title{
Restoration of miRNA-148a in pancreatic cancer reduces invasion and metastasis by inhibiting the Wnt/ $\beta$-catenin signaling pathway via downregulating maternally expressed gene-3
}

\author{
YUNPENG SUN, QIANDONG ZHU, MENGTAO ZHOU, WENJUN YANG, \\ HONGQI SHI, YUNFENG SHAN, QIYU ZHANG and FUXIANG YU \\ Department of Hepatobiliary Surgery, The First Affiliated Hospital of Wenzhou Medical University, \\ Wenzhou, Zhejiang 325015, P.R. China
}

Received July 7, 2016; Accepted July 16, 2018

DOI: $10.3892 /$ etm.2018.7026

\begin{abstract}
Various microRNAs (miRNA) have been recognized potential novel tumor markers and have a critical role in cancer development and progression. Recently, methylation of miRNA-148a was identified as a crucial biochemical process in the progression of cancer. However, its potential role and in pancreatic cancer as well as the underlying mechanisms have remained largely elusive. The present study investigated the potential antitumor effect of miR-148a as well as its impact on invasion and metastasis in pancreatic cancer. It was found that the expression of miRNA-148a and the potential predictive biomarker maternally expressed gene-3 (MEG-3) were obviously decreased in human pancreatic cancer tissues compared with those in adjacent non-tumorous tissues. Furthermore, miR-148a was found to be downregulated in pancreatic cancer cell lines compared with normal pancreatic cells through promoter methylation. An MTT assay and a clonogenic assay demonstrated that restoration of miRNA-148a inhibited the proliferation and colony formation of pancreatic cancer cells. In addition, miR-148a transduction led to the upregulation of MEG-3 expression and promoted apoptosis of pancreatic cancer cells. Western blot analysis revealed that transduction of miR-148a markedly decreased the expression levels of $\mathrm{C}$-myc, cyclin D1 and $\beta$-catenin in pancreatic cancer cells. Methylation of miR-148a not only decreased the endogenous $\beta$-catenin levels but also inhibited the nuclear translocation of $\beta$-catenin to delay cell cycle progression. Furthermore, ectopic miR-148a methylation inhibited pancreatic cancer cell migration and invasion via causing an
\end{abstract}

Correspondence to: Professor Fuxiang Yu, Department of Hepatobiliary Surgery, The First Affiliated Hospital of Wenzhou Medical University, 132 Fanhaixi Road, Wenzhou, Zhejiang 325015, P.R. China

E-mail: yufuxianghos@163.com

Key words: miR-148a, pancreatic cancer, Wnt/ $\beta$-catenin, MEG-3, methylation, metastasis upregulation of MEG-3 expression. Most importantly, ectopic overexpression of miR-148a in pancreatic cancer cells inhibited tumor formation in an animal experiment. Taken together, miR-148a methylation is a crucial regulatory process to inhibit the proliferation and invasion of pancreatic cancer cells, and transduction of miR-148a suppressed the proliferation of pancreatic cancer cells through negative regulation of the Wnt/ $\beta$-catenin signaling pathway. The findings of the present study suggested that miRNA-148a acts as a tumor suppressor in pancreatic cancer and may contribute to the development of novel treatments for pancreatic cancer.

\section{Introduction}

Pancreatic cancer is the fifth leading cause of cancer-associated mortalities in the world (1). The five-year survival rate of affected patients is poor $(<10 \%)$ and in the majority of cases, pancreatic cancer is considered unresectable with a median survival of 6-11 months $(2,3)$. The poor prognosis is mainly based on difficulties in diagnosing pancreatic cancer at early stages, while no curative treatments are currently available for patients diagnosed at the late stage $(4,5)$. Surgical treatment and anti-neoplastic therapies combined with neoadjuvant approaches are the most common treatments for patients with resectable non-metastatic pancreatic cancer $(6,7)$. Total resection combined with strategies encompassing primary chemotherapy and radiation is performed in $15-20 \%$ of patients with borderline resectable, non-metastatic disease, which appears to be promising (8). A previous study reviewed the treatments for pancreatic cancer, including chemotherapy, radiotherapy and/or neoadjuvant therapy, by performing a meta-analysis of the available data and summarized the toxicities and clinical benefits of these treatments (9). However, the survival rate of patients with pancreatic cancer remains poor due to rapid metastasis and malignant features of pancreatic carcinoma cells. Therefore, while considerable research effort has been made to unveil the pathogenesis, the molecular mechanisms underlying the metastasis and systemic progression of pancreatic carcinoma have remain to be fully elucidated (10).

Maternally expressed gene-3 (MEG-3) is homologous with the mouse maternally imprinted gene Gt12, which was identified 
as an imprinted gene first mapped on mouse distal chromosome 12 and human chromosome 14q (11). MEG-3 encodes a long non-coding RNA (lncRNA) that has been evidenced to be a tumor suppressor whose downregulation is associated with the progression of various cancer types (12). Previous studies have indicated that MEG-3 functions through interacting with cyclic adenosine monophosphate, murine double minute $2 / \mathrm{p} 53$ and growth differentiation factor 15 to regulate tumor cell proliferation and metastasis $(13,14)$. Previous studies have also demonstrated that MEG-3 is downregulated in most of human cancer cell types, including melanoma, non-small cell lung cancer, meningioma, melanoma, colon cancer, leukemia, nasopharyngeal carcinoma and pancreatic cancer, while it is highly expressed in normal human tissue $(13,15,16)$. In addition, a crosstalk interaction between MEG-3 and tumor suppressor p53 signaling pathway has been reported in the regulation of the growth of testicular germ cell tumors (17). However, the molecular mechanisms of MEG-3 in pancreatic cancer have remained to be fully elucidated.

Small non-coding RNA (ncRNAs) have an essential role in tumor progression $(18,19)$. They are a class of functional RNA molecules in the regulation of growth, aggressiveness, apoptosis and prognosis as well as immunoregulatory function in patients with cancer (20-22). These functional RNA molecules include small nucleolar RNAs, ribosomal RNAs, microRNAs (miRNAs/miRs), transfer RNAs, Piwi-interacting RNAs and lncRNAs $(23,24)$. Aberration of ncRNA levels is thought to have a critical role in cancer-associated cellular physiological processes (25). Furthermore, miRNAs exert significant regulatory functions in carcinogenesis, growth and invasion via regulating their upstream and downstream molecules (26).

The present study investigated the association between the methylation of the gene encoding miR-148a and its expression in pancreatic cancer cells. The inhibitory effects and molecular mechanisms of miR-148a and MEG-3 in human pancreatic cancer cell lines were examined. Activation of miR-148a methylation was found to inhibit tumor formation, proliferation and invasion of pancreatic cancer cells in vitro as well as in vivo. miR-148a transduction is likely to induce MEG-3 via regulation of the $\mathrm{Wnt} / \beta$-catenin signaling pathway. The results clarified the association between miR-148a and MEG-3 in pancreatic cancer, which may contribute to the development of novel treatments for pancreatic cancer.

\section{Materials and methods}

Ethics statement. The present study was performed in strict accordance with the recommendations in the Guide for the Care and Use of Laboratory Animals of the National Institutes of Health. During experimental procedures and euthanasia, all efforts were made to minimize suffering. This study was approved by the Institutional Review Board and Ethics Committee of Wenzhou Medical University (Wenzhou, China; approval no. 0812-10421C4).

Cells and reagents. PANC-1 and Aspc-1 pancreatic cancer cell lines were purchased from the American Type Tissue Collection (Manassas, VA, USA) and were cultured in Dulbecco's modified Eagle's medium (DMEM; Invitrogen; Thermo Fisher Scientific, Inc., Waltham, MA, USA) with
$10 \%$ fetal bovine serum (FBS; Invitrogen; Thermo Fisher Scientific, Inc.). Human normal pancreatic cells (human primary pancreatic microvascular endothelial cells; Hengfei Bioscience, Shanghai, China; https://boyaobio.biomart.cn) were cultured in minimum essential medium (Thermo Fisher Scientific, Inc.) with $10 \%$ FBS. All cells were maintained at $37^{\circ} \mathrm{C}$ in a humidified atmosphere containing $5 \% \mathrm{CO}_{2}$. Pancreatic tumor and adjacent normal tissues from a patient (female, 43 years, recruited in March 2014) were obtained from the First Affiliated Hospital of Wenzhou Medical University (Wenzhou, China). Patient consent was obtained.

Transduction of lentivirus in pancreatic cancer cell lines. A mixture of pWPXL-miR-148a $(5 \mu \mathrm{g})$ or pWPXL-control (5 $\mu \mathrm{g}$ ) plasmids (Invitrogen; Thermo Fisher Scientific, Inc.) were transfected into PANC-1 or Aspc-1 cells using Lipofectamine 2000 reagent (Thermo Fisher Scientific, Inc.) to generate cell lines stably transfected with miR-148a expression or control vector. PANC-1 or Aspc-1 cells were transfected with the recombinant lentivirus-transducing units plus $6 \mathrm{mg} / \mathrm{ml}$ Polybrene (Sigma-Aldrich; Merck KGaA, Darmstadt, Germany) according to a previous protocol (27). PANC-1 and Aspc-1 cells were further transfected with pWPXL-miR-148a (5 $\mu \mathrm{g})$ in combination with G-A-T-A-base (GATA)-1, GATA2, catalytic domain protein-C region (CDP-CR) or upstream stimulatory factor 1 (USF1; all $100 \mathrm{ng}$; all Promega Corporation, Madison, WI, USA) as an internal control. At $72 \mathrm{~h}$ following transfection, a dual luciferase assay kit (0000060417; Promega Corporation) was used to measure luciferase activities according to manufacturer's instructions. Results were normalized to Renilla.

MTT cytotoxicity assay. PANC-1 and Aspc-1 cells were transfected with pWPXL-miR-148a or pWPXL-control in 96-well plates for $96 \mathrm{~h}$ in triplicate for each condition. Subsequently, $20 \mu \mathrm{l}$ MTT ( $5 \mathrm{mg} / \mathrm{ml}$; Sigma-Aldrich; Merck $\mathrm{KGaA}$ ) in PBS was added to each well and the cells were further incubated for $4 \mathrm{~h}$. The entire medium was removed and $100 \mu \mathrm{l}$ dimethyl sulfoxide (Sigma-Aldrich; Merck KGaA) was added to the wells to solubilize the crystals. The optical density was measured using an ELISA reader (Bio-Rad Laboratories, Inc., Hercules, CA, USA) at a wavelength of $450 \mathrm{~nm}$.

Colony formation assay. For each group, $4 \mathrm{ml}$ DMEM containing $5 \times 10^{4}$ cells $/ \mathrm{ml}$ was added to a 6 -well plate. Following culturing at $37^{\circ} \mathrm{C}$ at $5 \% \mathrm{CO}_{2}$ for 14 days, the supernatant was discarded and cells were washed with PBS (3X). Cells were then fixed with $4 \%$ paraformaldehyde for $15 \mathrm{~min}$ at room temperature. Colonies were counted under an inverted microscope (magnification, x40; Nikon Corporation, Tokyo, Japan).

Tumor cell migration and invasion assays. PANC-1 and Aspc-1 cells transfected with pWPXL-miR-148a or pWPXL-control for $48 \mathrm{~h}$. For the invasion assay, PANC-1 and Aspc-1 (5x10 in $200 \mu \mathrm{l}$ serum-free DMEM) were added to the upper chambers of BD BioCoat Matrigel Invasion Chambers (BD Biosciences, Franklin Lakes, NJ, USA) according to the manufacturer's instructions. For the migration assay, a control insert (BD Biosciences) was used instead of the Matrigel 
Invasion Chamber. Cells were incubated for $48 \mathrm{~h}$ at $37^{\circ} \mathrm{C}$. The tumor cell invasion and migration were determined in at least three randomly selected microscopic fields of the stained lower side of each membrane.

Flow cytometric analysis. An Annexin V-fluorescein isothiocyanate (FITC) and propidium iodide (PI) analysis system apoptosis detection kit (BD Biosciences) was used to evaluate apoptosis of PANC-1 and Aspc-1 cells. Cells were incubated with pWPXL-miR-148a or pWPXL-control for $12 \mathrm{~h}$ at $37^{\circ} \mathrm{C}$ and then collected. A total of $1 \times 10^{6}$ cells were suspended in binding buffer containing Annexin V-FITC and PI according to the manufacturer's instructions. Fluorescence was detected with a BD FACScan flow cytometer and analyzed using BD FACSChorus $^{\mathrm{TM}}$ Software 1.2 (BD Biosciences).

Immunohistochemical staining. Immunohistochemical staining was performed via the avidin-biotin-peroxidase technique. Paraffin-embedded tumor tissue sections were prepared. Sections were deparaffinized and rehydrated with xylene, alcohol and tap water for $1 \mathrm{~h}$ at $37^{\circ} \mathrm{C}$ in each step. Tissue sections were then incubated with hydrogen peroxide $(3 \%)$ for 10-15 min and subsequently blocked with a regular blocking solution for $10-15 \mathrm{~min}$ at $37^{\circ} \mathrm{C}$. Finally, the sections were incubated with anti-miR-148a (cat. no. 10302) or anti-MEG-3 (cat. no. 5122; all 1:500; Cell Signaling Technology, Inc., Danvers, MA, USA) antibody at $4^{\circ} \mathrm{C}$ for $12 \mathrm{~h}$. All sections were washed three times and incubated with horseradish peroxidase-conjugated goat anti-mouse secondary antibody (1:2,000; ab6785; Abcam, Cambridge, UK) for $1 \mathrm{~h}$ at $37^{\circ} \mathrm{C}$. An enhanced chemiluminescence substrate ECL Select ${ }^{\mathrm{TM}}$ (Bio-Rad Laboratories, Inc.) was used to detect protein expression. The results were observed using a fluorescent microscope (magnification, $\mathrm{x} 40$ ) and analyzed using Quantity One software version 4.62 (Bio-Rad Laboratories, Inc.).

Methylation regions of miR-148a in pancreatic cancer. The methylation rate of miRNA-148a in PANC-1 and Aspc-1 cells was determined by quantitative methylation-specific polymerase chain reaction (qMS-PCR). DNA was obtained from PANC-1, Aspc-1 and human normal pancreatic cells using the PicoPure $^{\text {TM }}$ DNA Extraction kit (Applied Biosystems; Thermo Fisher Scientific, Inc.). A total of $10 \mathrm{ng}$ of DNA was amplified using the AmpFLSTR ${ }^{\mathrm{TM}}$ SGM Plus ${ }^{\mathrm{TM}}$ PCR Amplification kit (Thermo Fisher Scientific, Inc.) with the following primers: miRNA-148a, forward, 5'-TGGGTATTTGTTTTTGTTGAT TG-3' and reverse, 5'-ACTACACTTA AACCCCCTCTA ACC-3'. Reactions were performed using the following conditions: $90^{\circ} \mathrm{C}$ for $2 \mathrm{~min}$, followed by 40 cycles of $95^{\circ} \mathrm{C}$ for $20 \mathrm{sec}$, $60^{\circ} \mathrm{C}$ for $30 \mathrm{sec}$ and $72^{\circ} \mathrm{C}$ for $15 \mathrm{sec}$ and a final extension of $10 \mathrm{~min}$ at $72^{\circ} \mathrm{C}$. PCR products were diluted with water (1:500) and $1 \mu \mathrm{l}$ diluted product was subjected to qMS-PCR using LightCycler ${ }^{\circledR} 480$ SYBR-Green I Master mix (Thermo Fisher Scientific, Inc.). Reverse transcription-qPCR was performed using the High Capacity cDNA Reverse Transcription kit (Invitrogen; Thermo Fisher Scientific, Inc.) according to manufacturer's instructions. Primers were designed as follows: Unmethylated-specific miRNA-148a forward, 5'-TAT GATTTGTTTTATTATTGGTT-3' and reverse, 5'-AAC ACTAACAACATCAACAACC-3'; methylated-specific
miRNA-148a forward, 5'-TGATTCGTT TTATTATCG GTC-3' and reverse, 5'-AACACTAACGACATCGACG-3'; and $\beta$-actin forward, 5'-ACGGTCAGGTCATCACTATCG-3' and reverse, 5'-GGCATAGAGGTCTTTACGGATG-3'. The reaction was conducted at $95^{\circ} \mathrm{C}$ for $10 \mathrm{~min}$ followed by 40 cycles of $95^{\circ} \mathrm{C}$ for $15 \mathrm{sec}$ and $60^{\circ} \mathrm{C}$ for $60 \mathrm{sec}$. The methylation level was using the $2^{-\Delta \Delta \mathrm{Cq}}$ method (28). The results are expressed as the fold of the $\beta$-actin.

5-aza-2'-deoxycytidine treatment of PANC-1 and Aspc-1. For treatment with DNA methylation inhibitor 5-aza2'-deoxycytidine (5-aza-dC; 10 pM in PBS; Sigma-Aldrich; Merck KGaA), PANC-1 and Aspc-1 cells (1x10 $\left.{ }^{5}\right)$ were seeded into 6-well plates on day 0 and exposed to 5 -aza-dC at a final concentration of $5 \mu \mathrm{mol} / 1$ for 2 days at $37^{\circ} \mathrm{C}$. Control samples were incubated with an equal volume of PBS. Cells were harvested for RT-qPCR to assess miRNA-148a expression as described above.

Cell lines stably expressing MEG3. To obtain a cell line stably expressing MEG3, the pcDNA3.0-MEG3 (Thermo Fisher Scientific, Inc.) expression vector was transfected into PANC-1 and Aspc-1 cells as described above. Cells were used for further analysis after $48 \mathrm{~h}$ transfection. Cells were screened with G418 (1,000 $\mu \mathrm{g} / \mathrm{ml}$, Sigma-Aldrich; Merck KGaA) as described previously (29). RT-qPCR was performed to examine the expression of MEG3 at the RNA level as described above. The cell line with stably expressing empty vector pcDNA3.0 was used as control.

Luciferase assays. 3'-UTR sequences of miR-148a target genes containing upstream and downstream $100 \mathrm{bp}$ flanking sequences of the putative miR148a target sites were amplified as described above using the AmpFLSTR ${ }^{\mathrm{TM}}$ SGM Plus ${ }^{\mathrm{TM}}$ PCR Amplification kit (Thermo Fisher Scientific, Inc.) from PANC-1 and Aspc-1 cells genomic DNA by PCR and cloned into the pMIR-Report-Vector (Ambion, USA). Primers used for PCR were as follow: Forward, 5'-TGGGTATTTGTTTTTGTTGATTG-3' and reverse, 5'-ACT ACACTTAAACCCCCTCTAACC-3'. 3'-UTR luciferase reporter assays were performed by calcium phosphate transient transfection $(100 \mathrm{pM})$ of PANC-1 and Aspc-1 cells $\left(1 \times 10^{6}\right)$ with $20 \mathrm{ng}$ of pMIR-reporter-3'-UTR and $200 \mathrm{ng}$ of the vector control or miR-148a expression clone and $10 \mathrm{ng}$ pCMV-Renilla (internal control). The Dual Luciferase assay (Promega Corporation, Madison, WI, USA) was used to measure the luciferase activities after 72-h transfection. All luciferase activities were normalized to Renilla and the ratio is presented.

Confocal laser microscopy. PANC-1 and Aspc-1 cells grown on lysine-coated glass coverslips were treated with pWPXL-miR-148a or pWPXL-control for 48 h. Subsequently, the cells were fixed with $4 \%$ paraformaldehyde, followed by blocking in $1 \%$ bovine serum albumin and $0.1 \%$ Triton X-100 in PBS for $60 \mathrm{~min}$ at $37^{\circ} \mathrm{C}$. The pancreatic tumor cells were then incubated with antibodies against MEG-3 (1:500 dilution; Cell Signaling Technology, Inc., Danvers, MA, USA) for $2 \mathrm{~h}$ at $25^{\circ} \mathrm{C}$ in a humidified atmosphere. Subsequently, the cells were incubated with Alexa Fluor 488-conjugated anti-rabbit secondary antibody (1:400 dilution; cat. no.4412; Cell Signaling Technology, Inc.) after washing with PBS. Pancreatic tumor 
cell nuclei were stained with DAPI $(10 \mathrm{mg} / \mathrm{ml})$ for $30 \mathrm{~min}$ at $25^{\circ} \mathrm{C}$ in a humidified atmosphere. The PANC-1 and Aspc-1 cells were mounted in anti-fade mounting medium and images were captured using a Zeiss Confocal Spectral microscope (magnification, x40; Carl Zeiss, Jena, Germany).

Western blot analysis. PANC-1 and Aspc-1 cells were treated with pWPXL-miR-148a or pWPXL-control for $24 \mathrm{~h}$ at $37^{\circ} \mathrm{C}$, homogenized in lysate buffer containing protease-inhibitor and centrifuged at $6,000 \mathrm{x} \mathrm{g}$ at $4^{\circ} \mathrm{C}$ for $10 \mathrm{~min}$. The supernatant of was used for analysis of the total protein concentration using bicinchoninic acid protein assay kit (Thermo Fisher Scientific, Inc.). For detection, proteins $(40 \mu \mathrm{g})$ were loaded and separated using $12 \%$ SDS-PAGE gels, transferred to nitrocellulose membranes and hybridized as previously described (30). Subsequent to blocking in 5\% skimmed milk, membranes were probed with primary antibodies MEG-3 (1:500; 5122 Cell Signaling Technology, Inc.), E-cadherin (1:1,000; ab11512), Vimentin (1:1,000; ab92547), Snail2 (1:1,000; ab180714), $\beta$-catenin $(1: 1,000 ;$ ab32572), C-myc (1:1,000; ab32072), Cyclin D1 (1:1,000; ab134175) and $\beta$-actin $\left(1: 1,000\right.$; ab8226) and incubated for $1 \mathrm{~h}$ at $37^{\circ} \mathrm{C}$, followed by incubation with HRP-conjugated goat anti-mouse secondary antibody $(1: 2,000$; ab6785; all Abcam) for $24 \mathrm{~h}$ at $4^{\circ} \mathrm{C}$. The blots were visualized using a chemiluminescence kit (Thermo Fisher Scientific, Inc.). Quantity of protein was analyzed using Quantity One software version 4.62 (Bio-Rad Laboratories, Inc.).

Pancreatic colonization assay of PANC-1-miR-148 a cells in nude mice. Six-week-old female BALB/c nude mice $(\mathrm{n}=40$; weight, 20-25 g) were purchased from Beijing Vital River Laboratory Animal Technology Co., Ltd (Beijing, China). All animals were reared under specific pathogen-free conditions. Mice were maintained at a 12-h light/dark cycle with free access to food and water. Two million PANC-1-miR-148a or PANC-1-control cells were injected into the right flank of female $\mathrm{BALB} / \mathrm{c}$ nude mice $(\mathrm{n}=20$ randomized mice/group). On day 25 , tumors were isolated from experimental animals and used for further analysis. Tumor diameters were recorded once every two days and the tumor volume was calculated by using the following formula: $0.52 \mathrm{x}$ smallest diameter $\mathrm{x} 2 \mathrm{x}$ largest diameter.

Statistical analysis. Values are expressed as the mean \pm standard error of the mean. Data were analyzed using SPSS 1.0 software (SPSS, Chicago, IL, USA). Comparisons of data between multiple groups were analyzed by analysis of variance followed by Tukey's post-hoc test. $\mathrm{P}<0.05$ was considered to indicate a statistically significant difference.

\section{Results}

Downregulation of miR-148a and MEG-3 in pancreatic cancer. To explore the role of miR-148a and MEG-3 in pancreatic cancer, tissue samples from patients and cell lines were assessed by RT-qPCR and immunohistochemical analysis. Compared with normal adjacent tissues and a normal pancreatic cell line, miR-148a and MEG-3 were significantly decreased in pancreatic cancer tissues and cell lines (Fig. 1A-D). Overall, these results indicated that miR-148a and MEG-3 were downregulated in pancreatic cancer cell lines and tumors.

Identification of differential methylation regions of miR-148a in pancreatic cancer. To investigate of differential methylation regions of miR-148a in pancreatic cancer, an analysis of the 2,000-bp upstream DNA sequence of pre-miR-148a and a qMS-PCR were performed to confirm the extent of methylation and transcription factor binding sites. As shown in Fig. 2A, 5 putative $\mathrm{CpG}$ islands in the promoter of the gene encoding miR-148a were confirmed in PANC-1 and 4 putative $\mathrm{CpG}$ islands in Aspc-1. As shown in Fig. 2B, these $\mathrm{CpG}$ islands were differentially methylated in 5-aza-2'-deoxycytidine-treated PANC-1 and Aspc-1 cells. As displayed in Fig. 2C, the CpG sites within -144 to +172 bp of the promoter of the gene encoding miR-148a in PANC-1 and Aspc-1 cells were differentially methylated compared to those in normal pancreatic cells. Luciferase assays revealed that in pancreatic cancer cell lines, the promoter activity of a luciferase reporter vector containing clones of catalytic domain protein- $\mathrm{C}$ region (CDP-CR) differential methylation region mutations was the same as that in normal pancreatic cells, but those of GATA 1/2 and USF1 mutant reporters were 40-60\% higher than those in normal pancreatic cells (Fig. 2D). In addition, the promoter activity of a luciferase reporter vector containing differential miR-148a methylation regions was higher after co-transfection of GATA1, GATA2 or USF1 compared with control (Fig. 2E). The results in Fig. 2F indicated that the binding affinity between USF1 and the methylated probe decreased, indicating that methylcytosines affected the transcription activator USF1. Overall, these results indicated that the methylation status in pancreatic tumor cells is high compared to that in normal pancreatic cells and that GATA1, GATA2 and USF1 are involved in the activation of differential methylation regions, suggesting that the decreased ability of USF1 to bind to the methylated DNA may be associated with the reduction of miR-148a transcription in pancreatic tumor cells.

Restoration ofmiR-148a suppresses cell growth and metastatic capacity of pancreatic cancer. After confirmation of the downregulation of miR-148a in pancreatic cancer, the present study further analyzed the association between miR-148a and pancreatic cancer metastasis. Subsequently, PANC-1 and Aspc-1 cell lines stably transfected with miR-148a expression vector were established by lentivirus infection. It was observed that miR-148a exerted obvious inhibitory effects on the growth of PANC-1 and Aspc-1 cells (Fig. 3A). In addition, restoration of miR-148a expression suppressed the migration and invasion of PANC-1 and Aspc-1 cells (Fig. 3B and C). Furthermore, colony formation assays demonstrated that the number and size of pancreatic metastasis nodules was markedly inhibited in PANC-1-miR-148a and Aspc-1-miR-148a cells compared to those in the miR-148a-vector control groups (Fig. 3D). Conversely, PANC-1 and Aspc-1 cells transfected with miR-148a showed higher endogenous miR-148a expression, resulting in increased pancreatic cancer cell apoptosis (Fig. 3E). In addition, miR-148a transfection in PANC-1 and Aspc-1 cells promoted MEG-3 expression (Fig. 3F). Collectively, the results suggested that restoration of miR-148a 

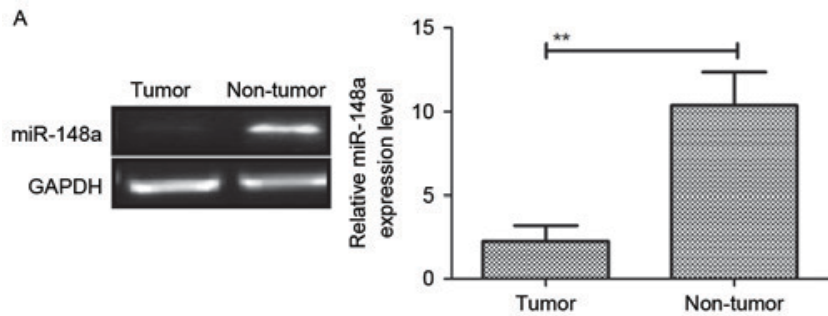

c
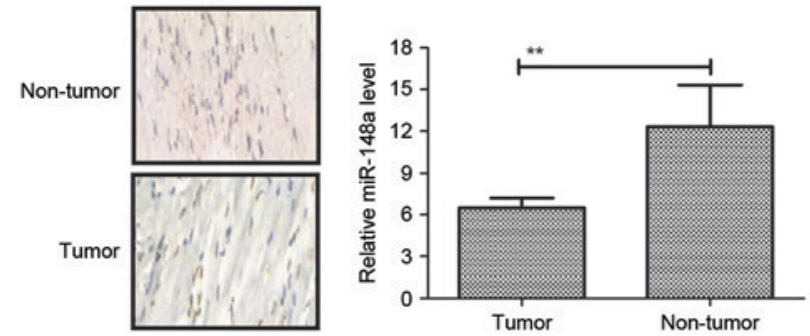

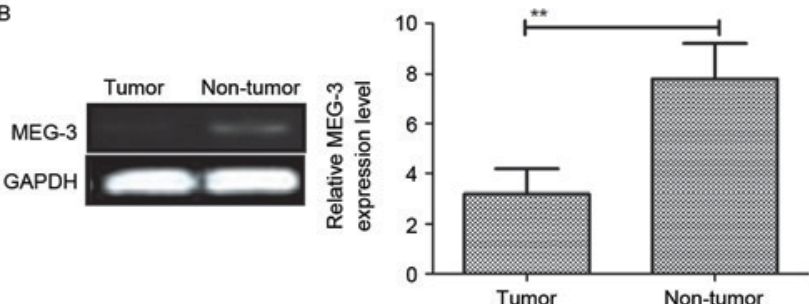

D
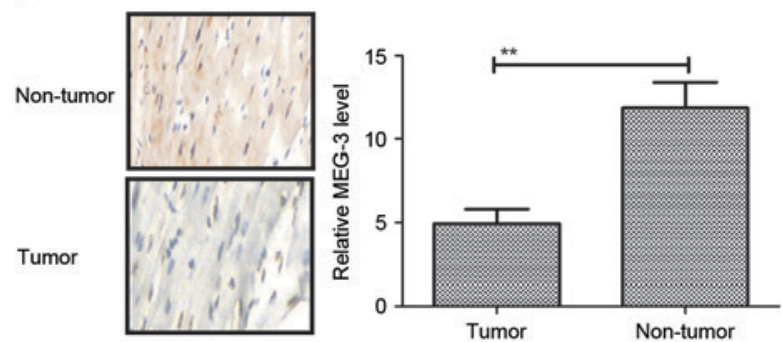

Figure 1. Expression levels of miR-148a and MEG-3 in pancreatic cancer tissues. Tumors obtained from patients with pancreatic cancer showed (A) higher miR-148a levels and (B) MEG-3 levels than those from normal control tissues. Representative gels of polymerase chain reaction products and quantified expression levels are displayed. (C and D) Immunofluorescence analysis of (C) miR-148a and (D) MEG-3 expression in tumors obtained from patients with pancreatic cancer and normal control tissues. Representative immunostaining images and quantified expression levels are displayed (magnification, $\mathrm{x} 40$ ). Values are expressed as the mean \pm standard error of the mean. ${ }^{* *} \mathrm{P}<0.01$ vs. control. MEG-3, maternally expressed gene-3; miR, microRNA.

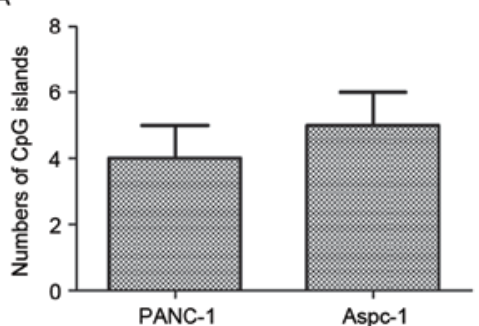

D

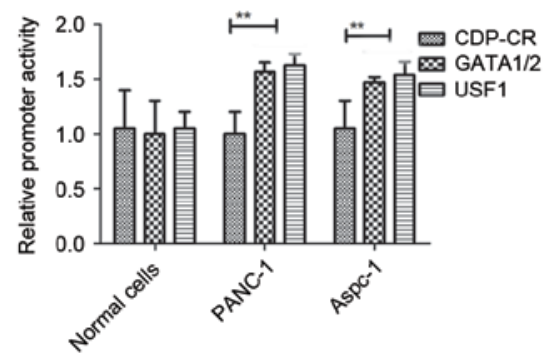

B

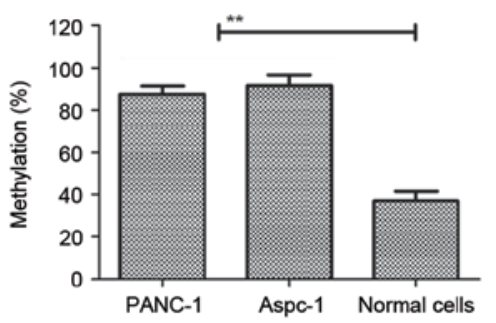

$E$
C

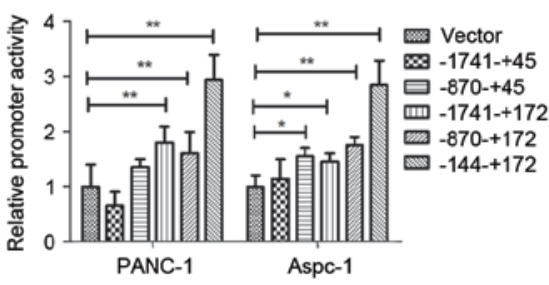

$\mathrm{F}$

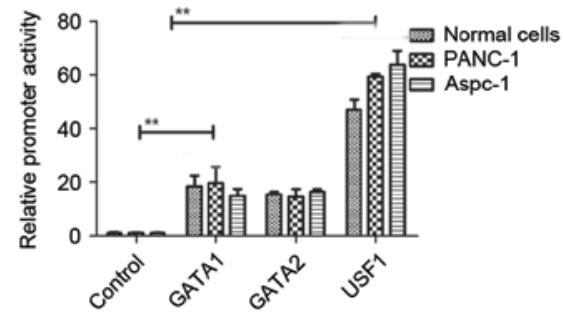

Figure 2. Identification of differential methylation status/regions of miR-148a in pancreatic cancer. (A) Number of CpG islands of miR-148a in PANC-1 and Aspc-1 cells. (B) The extent of methylation in 5-aza-2'-deoxycytidine-treated PANC-1 and Aspc-1 cells. (C) Identification of differential methylation regions of miR-148a in PANC-1 and Aspc-1 cells. (D) Promoter activity of a luciferase reporter vector containing clones of CDP-CR for differential methylation following transfection of PANC-1 and Aspc-1. Relative promoter activity of miR-148 clones with differential methylation regions. (E) Relative reporter activity containing different methylation region clones of miR-148a after co-transfection of GATA1, GATA2 or USF1. (F) An electrophoretic mobility shift assay was performed to analyze the miR-148a promoter containing the USF1 binding sequence treated with a methylated probe. Purified bacteria-recombinant USF1 protein was used as a mock control. Values are expressed as the mean \pm standard error of the mean. ${ }^{*} \mathrm{P}<0.05$ and ${ }^{* *} \mathrm{P}<0.01$ vs. control. miR, microRNA; USF1, upstream stimulatory factor 1; CpG, C-phosphate-G; CDP-CR, catalytic domain protein-C region; GATA, G-A-T-A base.

is a negative regulator inhibiting growth and metastatic potential in pancreatic cancer cells.

Restoration of miR-148a inhibits mesenchymal-epithelial transition (MET) through Wnt/ $\beta$-catenin signaling pathway mediated via MEG-3 in pancreatic cancer cells. Restoration of miR-148a led to inhibition of pancreatic cancer cells. In order to investigate the underlying molecular mechanisms and associated signal pathways, the present study assessed the effect of miR-148a on MET through the MEG-3-imediated 
A
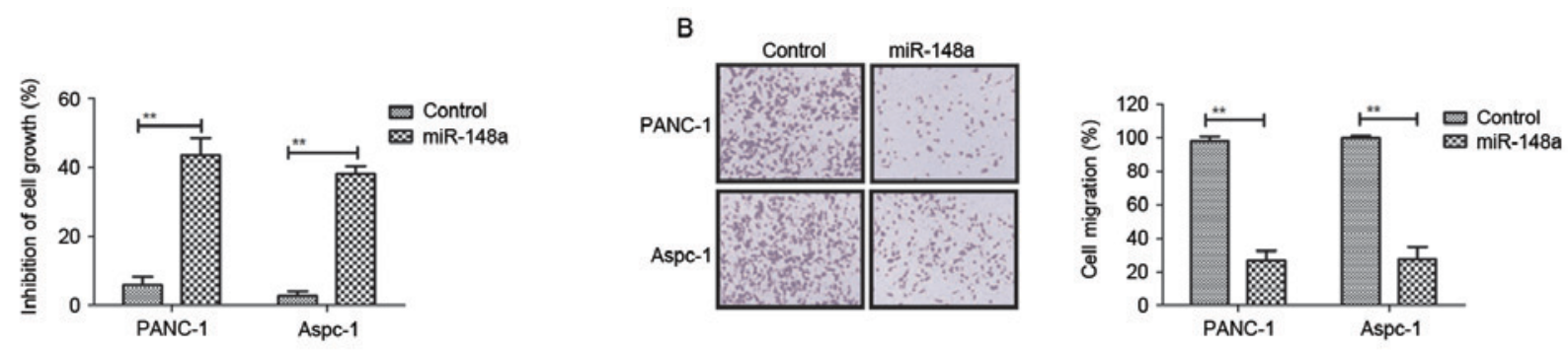

C
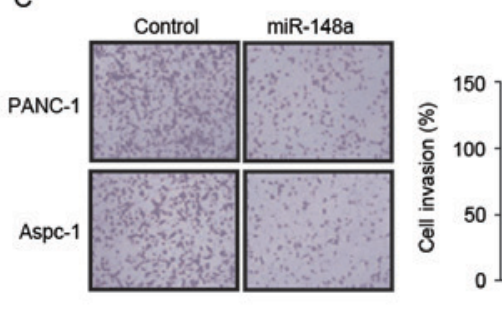

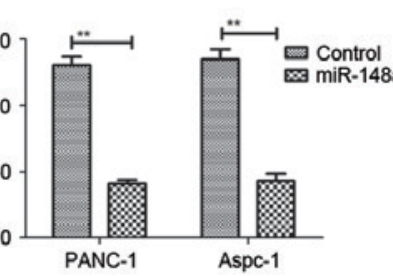

$E$
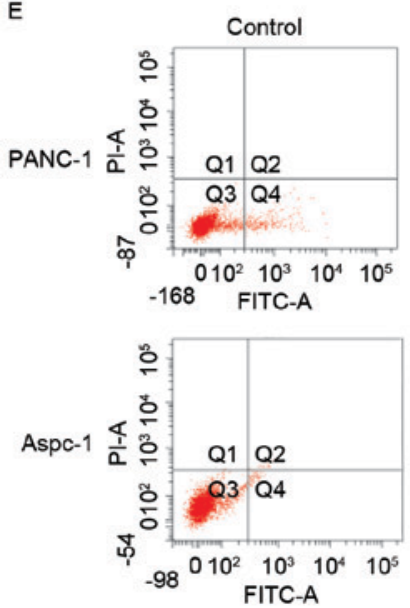

FITC-A
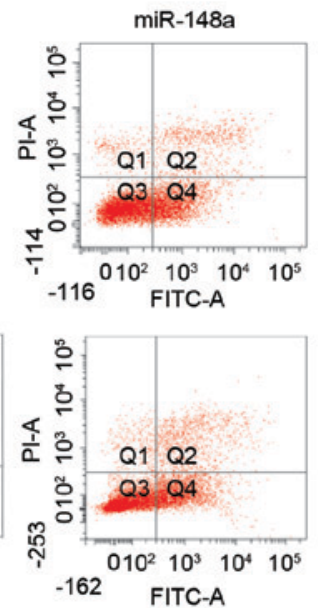
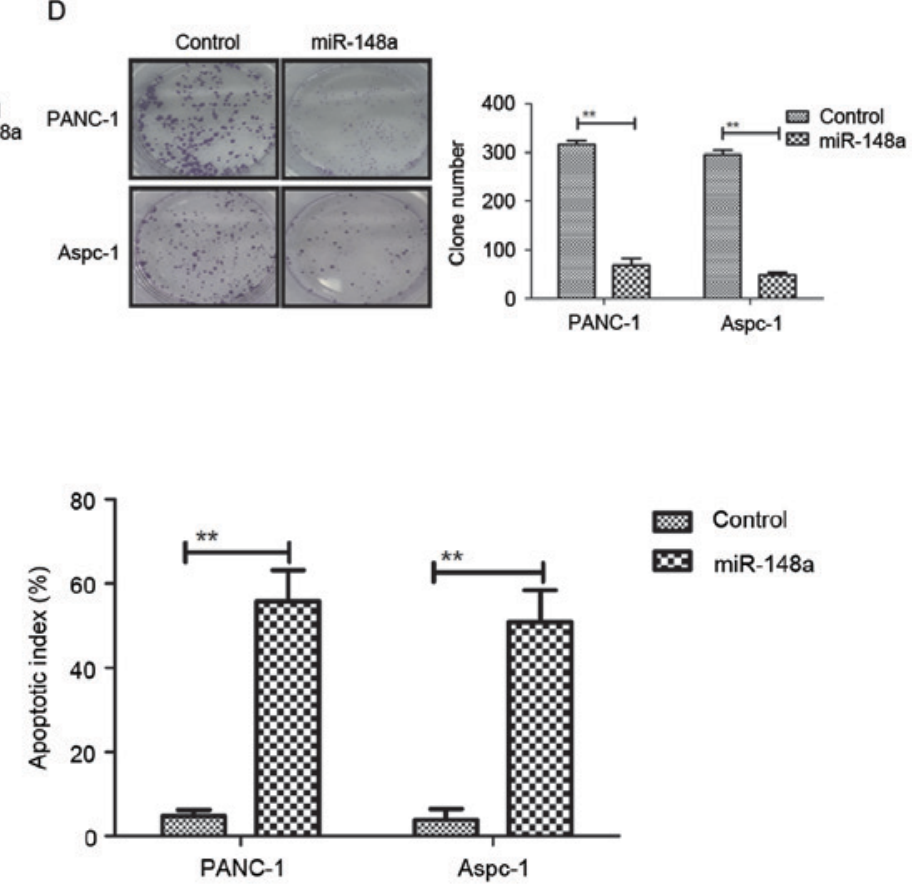

$\mathrm{F}$
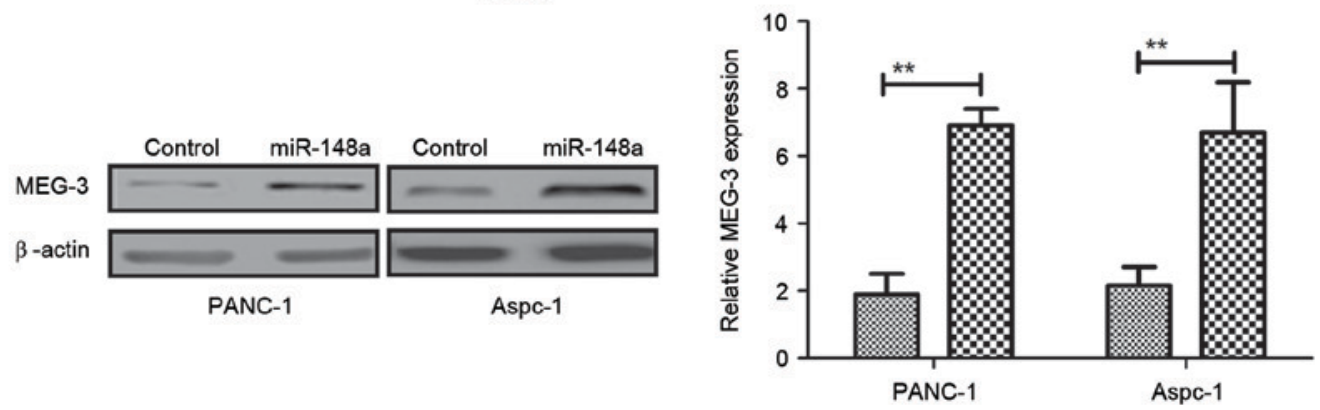

Figure 3. Inhibitory effects of miR-148a on growth and metastatic capacity of pancreatic cancer. (A) miR-148a exerted obvious inhibitory effects on growth of PANC-1 and Aspc-1 cells. (A-D) miR-148a exerted obvious inhibitory effects on (B) migration, (C) invasion and (D) colony formation by PANC-1 and Pasc-1 cells (magnification, $x 40$ ). (E and F) Transfection of miR-148a promoted (E) apoptosis and (F) MEG-3 expression of PANC-1 and Aspc-1 cells. Values are expressed as the mean \pm standard error of the mean. ${ }^{* *} \mathrm{P}<0.01$ vs. control. miR, microRNA; MEG-3, maternally expressed gene-3; FITC, fluorescein isothiocyanate; PI, propidium iodide.

Wnt/ß-catenin signaling pathway in pancreatic cancer cells. The results in Fig. 4A demonstrated that restoration of miR-148a markedly suppressed the expression of epithelial cell markers (E-cadherin, Vimentin and Snail2) in PANC-1 and Aspc-1 cells. In addition, restoration of miR-148a inhibited the expression of $\beta$-catenin, C-myc and Cyclin D1 in PANC-1 and Aspc-1 cells (Fig. 4B). A further experiment revealed that recombinant MEG-3 treatment also suppressed EMT and Wnt $/ \beta$-catenin signaling pathways in PANC-1 and Aspc-1 cells (Fig. 4C and D). In addition, restoration of miR-148a decreased endogenous $\beta$-catenin levels and suppressed the activity of nuclear translocation (Fig. 4E). Taken together, the results suggested that miR-148a regulates MEG-3 expression to activate the Wnt/ $\beta$-catenin signaling pathway to drive EMT in pancreatic cancer cells, which explains why the restoration of miR-148a inhibits cell growth and metastatic capacity of pancreatic cancer.

Restoration of miR-148a in pancreatic cancer cells leads to reduced tumor growth and invasion. To confirm the therapeutic 
A

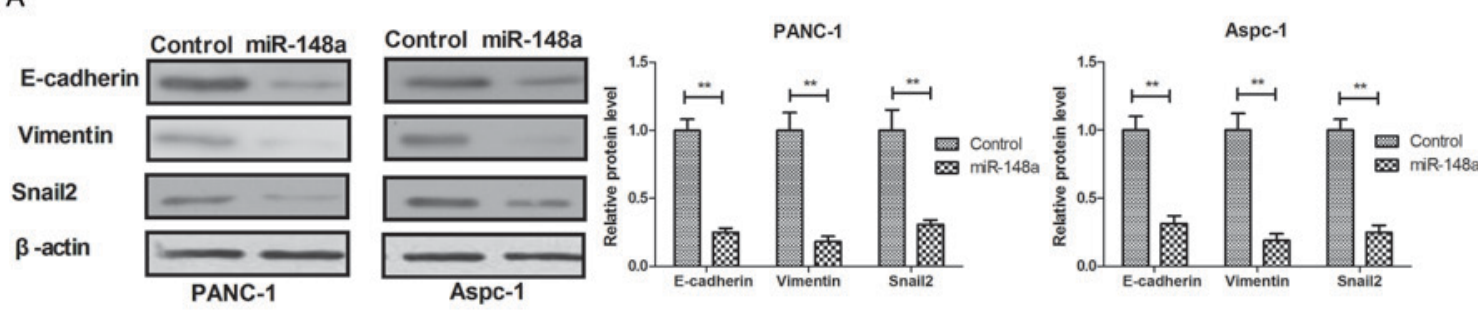

B
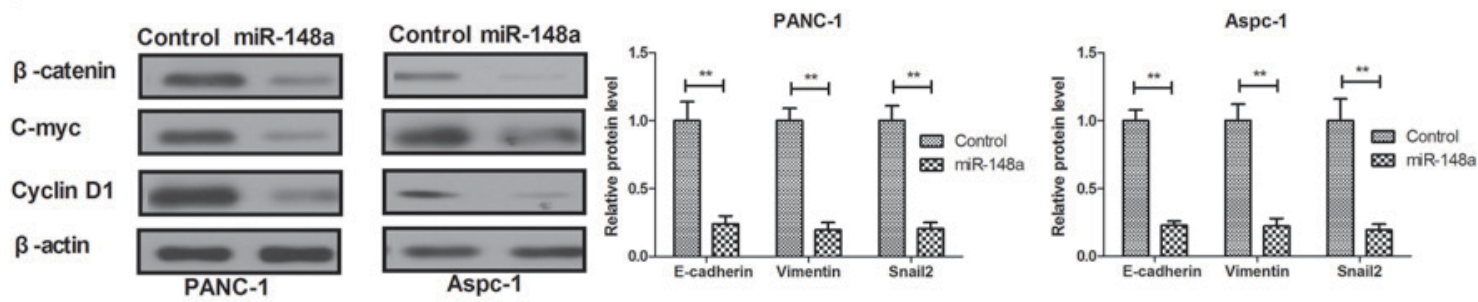

C
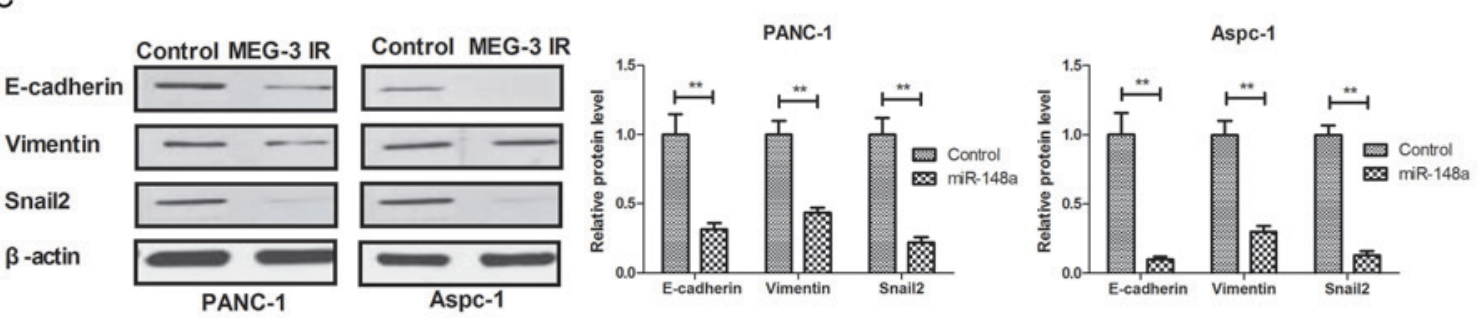

D
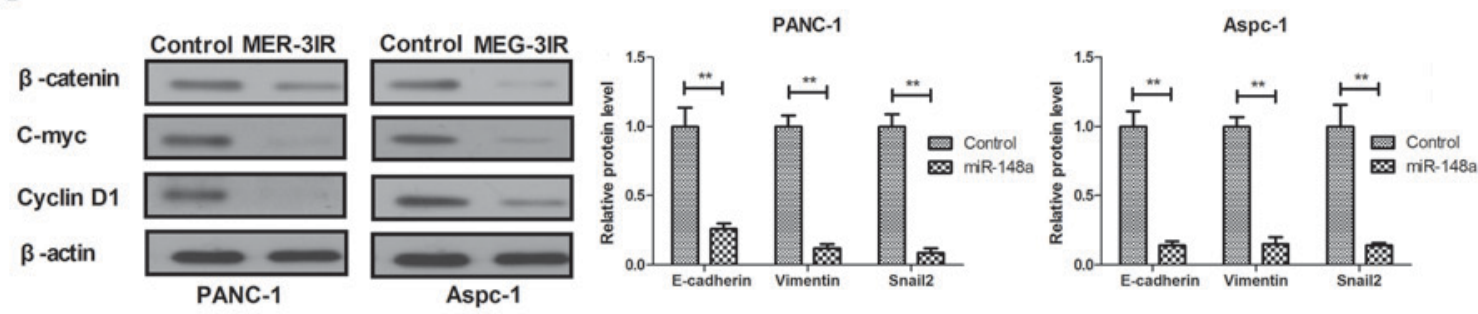

E
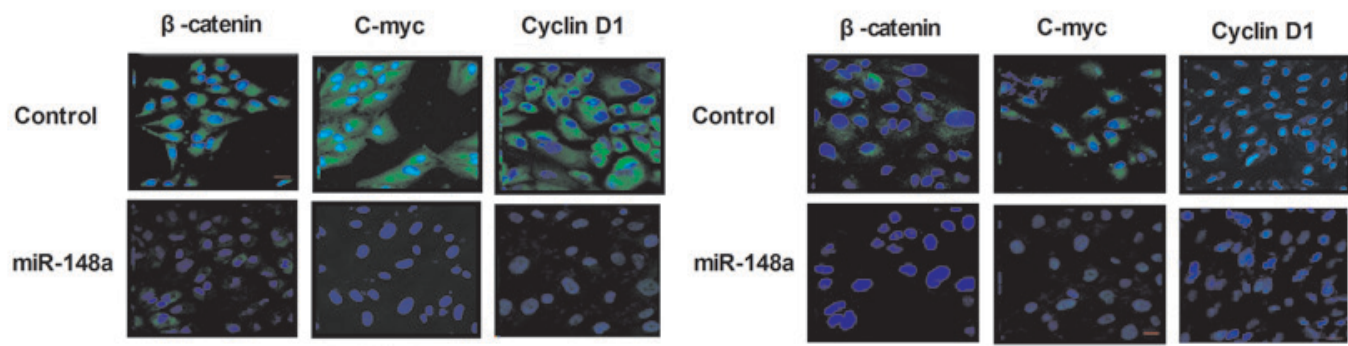

PANC-1

Aspc-1

Figure 4. Restoration of miR-148a inhibits mesenchymal-epithelial transition through MEG-3 mediated via the Wnt/ $\beta$-catenin signaling pathway in pancreatic cancer cells. Restoration of miR-148a (A) suppressed the expression of epithelial cell markers and (B) suppressed the expression of Wnt/ $\beta$-catenin signaling molecules in PANC-1 and Aspc-1 cells. (C and D) Recombinant MEG-3 inhibited $\beta$-catenin, C-myc and Cyclin D1 expression in (C) PANC-1 and (D) Aspc-1 cells. (E) Immunofluorescence microscopy revealed that restoration of miR-148a decreased endogenous $\beta$-catenin levels and suppressed the activity of nuclear translocation (magnification, $\mathrm{x} 40$ ). Nuclei were counterstained with DAPI. ${ }^{* *} \mathrm{P}<0.01 \mathrm{miR}$, microRNA; MEG-3, maternally expressed gene-3; IR, inhibitor; C-myc, c-myelocytomatosis oncogene.

effects of miR-148a restoration on cellular responses, the tumor growth in mice inoculated with PANC-1-miR-148a or PANC-1-miR-mimic (control) was assessed. The results in Fig. 5A showed that mice implanted with PANC-1-miR-148a hardly formed any tumor lesions, while those inoculated with PANC-1-vector formed obvious tumor tissue masses. In addition, histochemical analysis and RT-qPCR confirmed the overexpression of miR-148a in the tumors (Fig. 5B). To investigate the effects of miR-148a expression on the metastatic potential of PANC-1 cells, the total amount of pancreatic tumor nodules in each animal was evaluated. It was observed that PANC-1-miR-148a transfection decreased the amount of pancreatic tumor nodules in the mice harboring PANC-1 cells (Fig. 5C). Furthermore, the expression levels of the mediator MEG-3 and Wnt/ $\beta$-catenin signaling pathway proteins were assessed in the pancreatic cancer tissues in vivo. As shown in Fig. 5D, it was found that MEG-3 expression was upregulated, while $\beta$-catenin, $\mathrm{C}$-myc and Cyclin D1 expression levels 
A

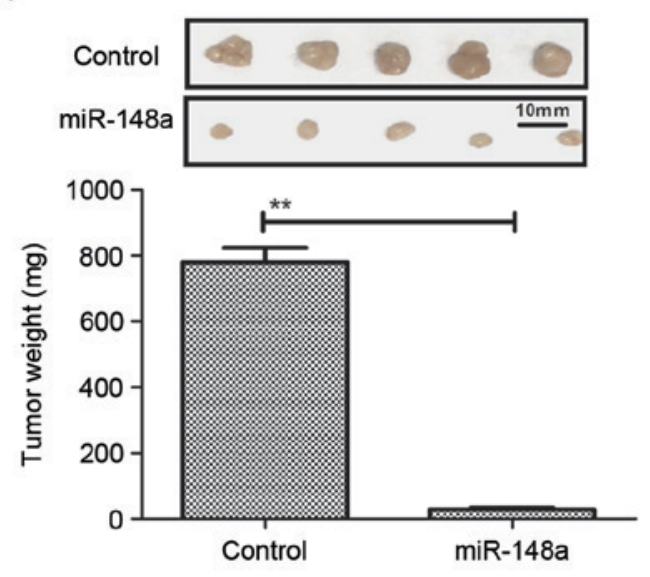

C

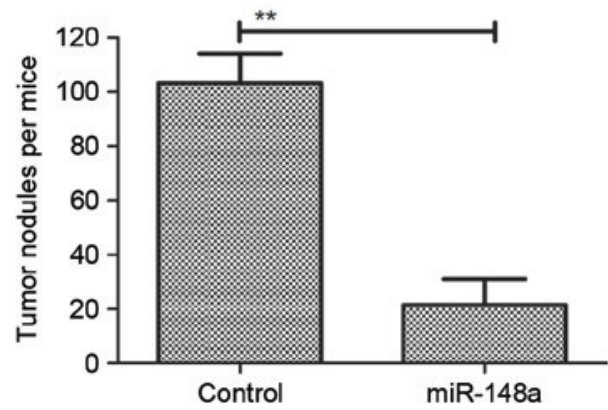

B
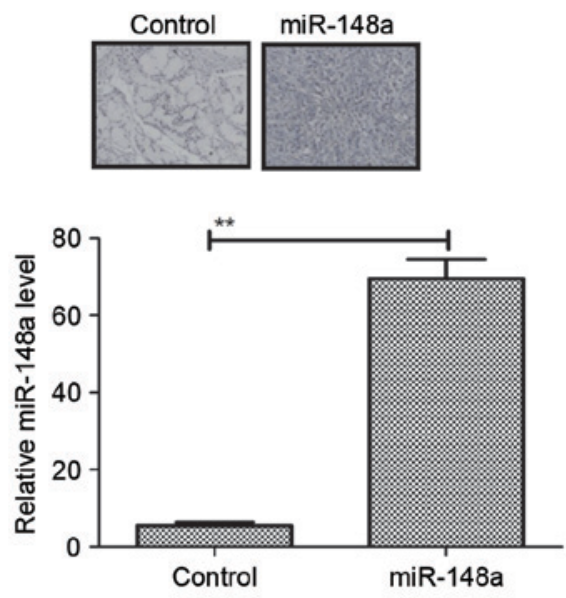

D

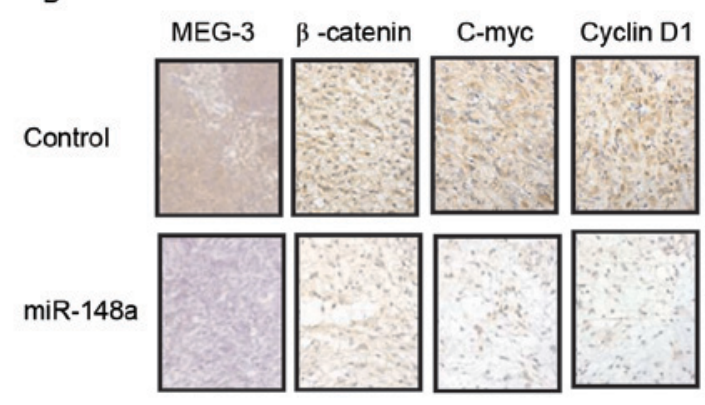

Figure 5. Reconstruction of miR-148a leads to reduced pancreatic tumor growth and invasion in vivo. (A) Implantation of PANC-1-miR-148a inhibited formation of pancreatic tumor lesions (scale bar, $10 \mathrm{~mm}$ ). (B) PANC-1-miR-148a showed higher miR-148a expression in vivo, as determined by histological analysis (magnification, x40). (C) Quantification of pancreatic tumor nodules within animals after injection of PANC-1-miR-148a or PANC-1-control cells. (D) Reconstruction of miR-148a decreased Wnt/ $\beta$-catenin expression levels by upregulating MEG-3 expression in vivo (magnification, $\mathrm{x} 40$ ). Values are expressed as the mean \pm standard error of the mean. ${ }^{* *} \mathrm{P}<0.01$ vs. control. miR, microRNA; MEG-3, maternally expressed gene-3.

were downregulated in tumors from mice implanted with PANC-1-miR-148a compared to those in the vector group. Collectively, these results indicated that miR-148a restoration in PANC-1 cells not only impaired Wnt/ $\beta$-catenin-induced cell growth and invasion via MEG-3 in vitro but also in vivo.

\section{Discussion}

A large number of studies have indicated that miRNAs constitute complex and efficient regulatory networks via post-transcriptional regulation in cellular biochemistry $(26,31)$. Recently, post-transcriptional regulation of miRNAs in various cancer cells has drawn the attention of scientists in the field of cancer therapy $(32,33)$. Aberrant miRNA expression in various tumor cell types has been evidenced to have important roles in carcinogenesis, tumorigenesis, tumor suppression and tumor progression (34-36). The present study investigated the function of miR-148a in pancreatic cancer. The results indicated that miR-148a is frequently downregulated in human pancreatic cancer cells and tissues and that its downregulation is significantly associated with growth, invasion and metastasis through regulating the MEG-3-induced Wnt/ $\beta$-catenin signaling pathway in pancreatic cancer. Further analysis revealed that restoration of miR-148a suppressed pancreatic carcinoma cell growth, colony formation, migration and invasion in vitro as well as tumor growth and metastasis in vivo.
The functional target molecule of miR-148a was identified as MEG-3 in pancreatic cancer cells and tumors. The results of the present study suggested that miR-148a is a potential tumor suppressor and inhibits metastasis of pancreatic cancer, and that its restoration may contribute to tumor regression in patients with pancreatic cancer.

Pancreatic cancer is a common malignancy worldwide and with the increasing rate of newly diagnosed cases, is bound to become the second leading cause of cancer-associated mortality in the future $(8,37)$. Based on the findings of previous studies, a better understanding of cancer pathology, earlier diagnosis and systemic therapies and the development of more efficacious drugs may improve the five-year survival rate of patients with pancreatic cancer (37-39). However, $>85 \%$ of patients are newly diagnosed with pancreatic cancer at the advanced stage, which contributes to the difficulty of their treatment and dismal prognosis (40). At present, treatment options for pancreatic cancer are based on chemotherapy and radiotherapy regimens. Although numerous studies, which have proposed different schedules to improve the overall survival and outcomes for patients with pancreatic cancer, are encouraging additional studies are still required to corroborate the molecular mechanisms $(1,41,42)$. The present study provided novel insight and potential therapeutic approaches for the treatment of pancreatic cancer, suggesting the potential application of miR-148a for the treatment of human cancers. 
Certain miRNAs are involved in tumorigenesis, progression and metastasis in the majority of cancer cells, and are potentially involved in the regulation of proliferation-associated cellular processes, including mitosis and chromosome replication (43). The present study indicated that miR-148a acts as a tumor suppressor in pancreatic cancer and it is downregulated through epigenetic silencing by promoter methylation. Although it has been reported that miR-148a silencing by hypermethylation activates the integrin-mediated signaling pathway in nasopharyngeal carcinoma, the detailed mechanisms of the biological roles of miR-148a have largely remained elusive (44). The present study identified that ectopic expression of the MEG-3 gene is controlled by miR-148a expression in human pancreatic cancer cells and tumors, and that MEG-3 overexpression significantly inhibited the proliferation and invasion for pancreatic cancer cells.

MEG-3 encodes a long non-coding RNA that has been recently shown to regulate tumorigenesis through its interaction with miRNA (13). Liu et al (45) assessed the expression levels and molecular mechanisms of the lncRNA-encoding gene MEG-3 in gallbladder cancer and the results indicated that pcDNA-MEG3-transfected cells reduced the activity and clone counts of gallbladder cancer cells and increased p53 and apoptosis. In addition, Luo et al (46) showed that overexpression of lncRNA MEG-3 inhibits cell proliferation and invasion, and induces apoptosis in prostate cancer in vitro as well as in vivo via reducing the protein expression of B-cell lymphoma 2 (Bcl-2), enhancing Bcl-2-associated X protein and activating caspase 3. Furthermore, Chunharojrith et al (17) indicated that pituitary tumor growth is suppressed by MEG-3 IncRNA, leading to cell cycle arrest at the G1 phase. The results of the present study revealed that overexpression of MEG-3 resulted in downregulation of the $\mathrm{Wnt} / \beta$-catenin signaling pathway in pancreatic cancer. Furthermore, inhibition of MEG-3 expression abrogated inhibitory effects from miR-148a restoration in pancreatic cancer cells.

In conclusion, the present study investigated the inhibitory effects of miRNA-148a in pancreatic cancer and demonstrated its anti-proliferative and anti-metastatic potential, which may be utilized for cancer treatment. Lower expression levels of miRNA-148a caused by DNA hypermethylation were associated with pancreatic tumor growth and aggressive behavior. The present study provided a potential rationale for developing epigenetic therapies through restoration of tumor-suppressor miRNA-148a to inhibit invasion and metastasis by downregulating the Wnt/ $\beta$-catenin signaling pathway through upregulating MEG-3 in pancreatic cancer. The results suggested that miRNA-148a may be a potential anti-cancer agent for the treatment of patients with pancreatic carcinoma to complement clinical cancer management strategies.

\section{Acknowledgements}

Not applicable.

\section{Funding}

The current study was supported by the Educational Department of Zhejiang Province (grant no. Y201534189).

\section{Availability of data and materials}

The analyzed data sets generated during the study are available from the corresponding author on reasonable request.

\section{Authors' contributions}

YSu performed the experiments. QZhu, MZ, WY, HS, YSh and QZha prepared and analyzed experimental data. FY designed the experiment. All authors read and approved the final version of the manuscript.

\section{Ethics approval and consent to participate}

The present study was performed in strict accordance with the recommendations in the Guide for the Care and Use of Laboratory Animals of the National Institutes of Health. This study was approved by the Institutional Review Board and Ethics Committee of Wenzhou Medical University (approval no. 0812-10421C4).

\section{Patient consent for publication}

Not applicable.

\section{Competing interests}

The authors declare that they have no competing interests.

\section{References}

1. Dumstrei K, Chen $\mathrm{H}$ and Brenner H: A systematic review of serum autoantibodies as biomarkers for pancreatic cancer detection. Oncotarget 7: 11151-11164, 2016.

2. Klompmaker S, de Rooij T, Korteweg JJ, van Dieren S, van Lienden KP, van Gulik TM, Busch OR and Besselink MG: Systematic review of outcomes after distal pancreatectomy with coeliac axis resection for locally advanced pancreatic cancer. $\mathrm{Br}$ J Surg 103: 941-949, 2016.

3. Paiella S, Sandini M, Gianotti L, Butturini G, Salvia R and Bassi C: The prognostic impact of para-aortic lymph node metastasis in pancreatic cancer: A systematic review and meta-analysis. Eur J Surg Oncol 42: 616-624, 2016.

4. Subramani R, Gangwani L, Nandy SB, Arumugam A, Chattopadhyay $\mathrm{M}$ and Lakshmanaswamy R: Emerging roles of microRNAs in pancreatic cancer diagnosis, therapy and prognosis (Review). Int J Oncol 47: 1203-1210, 2015.

5. Yang J, Li J, Zhu R, Zhang H, Zheng Y, Dai W, Wang F, Shen M, Chen K, Cheng P, et al: K-ras mutational status in cytohistological tissue as a molecular marker for the diagnosis of pancreatic cancer: A systematic review and meta-analysis. Dis Markers 2014: 573783, 2014.

6. Spadavecchia J, Movia D, Moore C, Maguire CM, Moustaoui H, Casale S, Volkov Y and Prina-Mello A: Targeted polyethylene glycol gold nanoparticles for the treatment of pancreatic cancer: From synthesis to proof-of-concept in vitro studies. Int J Nanomedicine 11: 791-822, 2016.

7. Goldsmith C, Price P, Cross T, Loughlin S, Cowley I and Plowman N: Dose-volume histogram analysis of stereotactic body radiotherapy treatment of pancreatic cancer: A focus on duodenal dose constraints. Semin Radiat Oncol 26: 149-156, 2016.

8. Spadi R, Brusa F, Ponzetti A, Chiappino I, Birocco N, Ciuffreda L and Satolli MA: Current therapeutic strategies for advanced pancreatic cancer: A review for clinicians. World J Clin Oncol 7: 27-43, 2016.

9. Tang K, Lu W, Qin W and Wu Y: Neoadjuvant therapy for patients with borderline resectable pancreatic cancer: A systematic review and meta-analysis of response and resection percentages. Pancreatology 16: 28-37, 2016. 
10. Blogowski W, Bodnarczuk T and Starzynska T: Concise review: Pancreatic cancer and bone marrow-derived stem cells. Stem Cells Transl Med 5: 938-945, 2016.

11. Miyoshi N, Wagatsuma H, Wakana S, Shiroishi T, Nomura M, Aisaka K, Kohda T, Surani MA, Kaneko-Ishino T and Ishino F: Identification of an imprinted gene, Meg3/Gt12 and its human homologue MEG3, first mapped on mouse distal chromosome 12 and human chromosome 14q. Genes Cells 5: 211-220, 2000.

12. Oczkowicz M, Ropka-Molik K, Piorkowska K, Rozycki M and Rejduch B: Frequency of DLK1 c.639C $>$ T polymorphism and the analysis of MEG3/DLK1/PEG11 cluster expression in muscle of swine raised in Poland. Meat Sci 88: 627-630, 2011.

13. Benetatos L, Vartholomatos G and Hatzimichael E: MEG3 imprinted gene contribution in tumorigenesis. Int J Cancer 129: 773-779, 2011.

14. Kagami M, O'Sullivan MJ, Green AJ, Watabe Y, Arisaka O, Masawa N, Matsuoka K, Fukami M, Matsubara K, Kato F, et al: The IG-DMR and the MEG3-DMR at human chromosome 14q32.2: Hierarchical interaction and distinct functional properties as imprinting control centers. PLoS Genet 6: e1000992, 2010

15. Zhang X, Rice K, Wang Y, Chen W, Zhong Y, Nakayama Y, Zhou Y and Klibanski A: Maternally expressed gene 3 (MEG3) noncoding ribonucleic acid: Isoform structure, expression, and functions. Endocrinology 151: 939-947, 2010

16. Zhang X, Zhou Y, Mehta KR, Danila DC, Scolavino S, Johnson SR and Klibanski A: A pituitary-derived MEG3 isoform functions as a growth suppressor in tumor cells. J Clin Endocrinol Metab 88: 5119-5126, 2003.

17. Chunharojrith P, Nakayama Y, Jiang X, Kery RE, Ma J, De La Hoz Ulloa CS, Zhang X, Zhou Y and Klibanski A: Tumor suppression by MEG3 lncRNA in a human pituitary tumor derived cell line. Mol Cell Endocrinol 416: 27-35, 2015.

18. Lv J, Qiu M, Xia W, Liu C, Xu Y, Wang J, Leng X, Huang S, Zhu R, Zhao M, et al: High expression of long non-coding RNA SBF2-AS1 promotes proliferation in non-small cell lung cancer. J Exp Clin Cancer Res 35: 75, 2016.

19. Costales MG, Rzuczek SG and Disney MD: Comparison of small molecules and oligonucleotides that target a toxic, non-coding RNA. Bioorg Med Chem Lett 26: 2605-2609, 2016.

20. Victoria Martinez B, Dhahbi JM,Nunez Lopez YO,Lamperska K, Golusinski P, Luczewski L, Kolenda T, Atamna H, Spindler SR, Golusinski W and Masternak MM: Circulating small non-coding RNA signature in head and neck squamous cell carcinoma. Oncotarget 6: 19246-19263, 2015.

21. Nowacki FC, Swain MT, Klychnikov OI, Niazi U, Ivens A, Quintana JF, Hensbergen PJ, Hokke CH, Buck AH and Hoffmann KF: Protein and small non-coding RNA-enriched extracellular vesicles are released by the pathogenic blood fluke Schistosoma mansoni. J Extracell Vesicles 4: 28665, 2015.

22. Marchand V and Branlant C: Quantification and quality control of a small non-coding RNA preparation. Methods Mol Biol 1296: $17-28,2015$.

23. Marti E and Estivill X: Small non-coding RNAs add complexity to the RNA pathogenic mechanisms in trinucleotide repeat expansion diseases. Front Mol Neurosci 6: 45, 2013.

24. Martens-Uzunova ES, Olvedy M and Jenster G: Beyond microRNA-novel RNAs derived from small non-coding RNA and their implication in cancer. Cancer Lett 340: 201-211, 2013.

25. Prensner JR and Chinnaiyan AM: The emergence of lncRNAs in cancer biology. Cancer Discov 1: 391-407, 2011.

26. Munagala R, Aqil F and Gupta RC: Exosomal miRNAs as biomarkers of recurrent lung cancer. Tumour Biol 37: 10703-10714, 2016.

27. Dimitrova DI, Yang X, Reichenbach NL, Karakasidis S, Sutton RE, Henderson EE, Rogers TJ and Suhadolnik RJ: Lentivirus-mediated transduction of PKR into CD34(+) hematopoietic stem cells inhibits HIV-1 replication in differentiated T cell progeny. J Interferon Cytokine Res 25: 345-360, 2005.

28. Livak KJ and Schmittgen TD: Analysis of relative gene expression data using real-time quantitative PCR and the 2(-Delta Delta C(T)) method. Methods 25: 402-408, 2001.
29. Yallop CA and Svendsen I: The effects of G418 on the growth and metabolism of recombinant mammalian cell lines. Cytotechnology 35: 101-114, 2001.

30. Wai-Hoe L, Wing-Seng L, Ismail Z and Lay-Harn G: SDS-PAGE-based quantitative assay for screening of kidney stone disease. Biol Proced Online 11: 145-160, 2009.

31. Seviour EG, Sehgal V, Lu Y, Luo Z, Moss T, Zhang F, Hill SM, Liu W, Maiti SN, Cooper L, et al: Functional proteomics identifies miRNAs to target a $\mathrm{p} 27 / \mathrm{Myc} /$ phospho- $\mathrm{Rb}$ signature in breast and ovarian cancer. Oncogene 35: 691-701, 2016.

32. Madhavan D, Peng C, Wallwiener M, Zucknick M, Nees J, Schott S, Rudolph A, Riethdorf S, Trumpp A, Pantel K, et al: Circulating miRNAs with prognostic value in metastatic breast cancer and for early detection of metastasis. Carcinogenesis 37: 461-470, 2016.

33. Tian W, Liu J, Pei B, Wang X, Guo Y and Yuan L: Identification of miRNAs and differentially expressed genes in early phase non-small cell lung cancer. Oncol Rep 35: 2171-2176, 2016.

34. Endzelins E, Melne V, Kalnina Z, Lietuvietis V, Riekstina U, Llorente A and Linē A: Diagnostic, prognostic and predictive value of cell-free miRNAs in prostate cancer: A systematic review. Mol Cancer 15: 41, 2016.

35. Masliah-Planchon J, Garinet S and Pasmant E: RAS-MAPK pathway epigenetic activation in cancer: miRNAs in action. Oncotarget 7: 38892-38907, 2016.

36. Mathe A, Scott RJ and Avery-Kiejda KA: MiRNAs and other epigenetic changes as biomarkers in triple negative breast cancer. Int J Mol Sci 16: 28347-28376, 2015.

37. Lee JC, Ahn S, Paik KH, Kim HW, Kang J, Kim J and Hwang JH: Clinical impact of neoadjuvant treatment in resectable pancreatic cancer: A systematic review and meta-analysis protocol. BMJ Open 6: e010491, 2016.

38. Kunk PR, Bauer TW, Slingluff CL and Rahma OE: From bench to bedside a comprehensive review of pancreatic cancer immunotherapy. J Immunother Cancer 4: 14, 2016.

39. Kristensen A, Vagnildhaug OM, Gronberg BH, Kaasa S, Laird B and Solheim TS: Does chemotherapy improve health-related quality of life in advanced pancreatic cancer? A systematic review. Crit Rev Oncol Hematol 99: 286-298, 2016.

40. Paiella S, Salvia R, Ramera M, Girelli R, Frigerio I, Giardino A Allegrini V and Bassi C: Local ablative strategies for ductal pancreatic cancer (Radiofrequency Ablation, Irreversible Electroporation): A review. Gastroenterol Res Pract 2016: 4508376, 2016

41. Gagliardi AR, Soong D and Gallinger S: Identifying factors influencing pancreatic cancer management to inform quality improvement efforts and future research: A scoping systematic review. Pancreas 45: 161-166, 2016

42. Fan Y, Hu J, Feng B, Wang W, Yao G, Zhai J and Li X: Increased risk of pancreatic cancer related to gallstones and cholecystectomy: A systematic review and Meta-analysis. Pancreas 45: 503-509, 2016

43. Stuopelyte K, Daniunaite K, Jankevicius F and Jarmalaite S: Detection of miRNAs in urine of prostate cancer patients. Medicina (Kaunas) 52: 116-124, 2016.

44. Li HP, Huang HY, Lai YR, Huang JX, Chang KP, Hsueh C and Chang YS: Silencing of miRNA-148a by hypermethylation activates the integrin-mediated signaling pathway in nasopharyngeal carcinoma. Oncotarget 5: 7610-7624, 2014.

45. Liu B, Shen ED, Liao MM, Hu YB, Wu K, Yang P, Zhou L and Chen WD: Expression and mechanisms of long non-coding RNA genes MEG3 and ANRIL in gallbladder cancer. Tumour Biol 37: 9875-9786, 2016

46. Luo G, Wang M, Wu X, Tao D, Xiao X, Wang L, Min F, Zeng $F$ and Jiang G: Long Non-coding RNA MEG3 inhibits cell proliferation and induces apoptosis in prostate cancer. Cell Physiol Biochem 37: 2209-2220, 2015.

This work is licensed under a Creative Commons Attribution-NonCommercial-NoDerivatives 4.0 International (CC BY-NC-ND 4.0) License. 